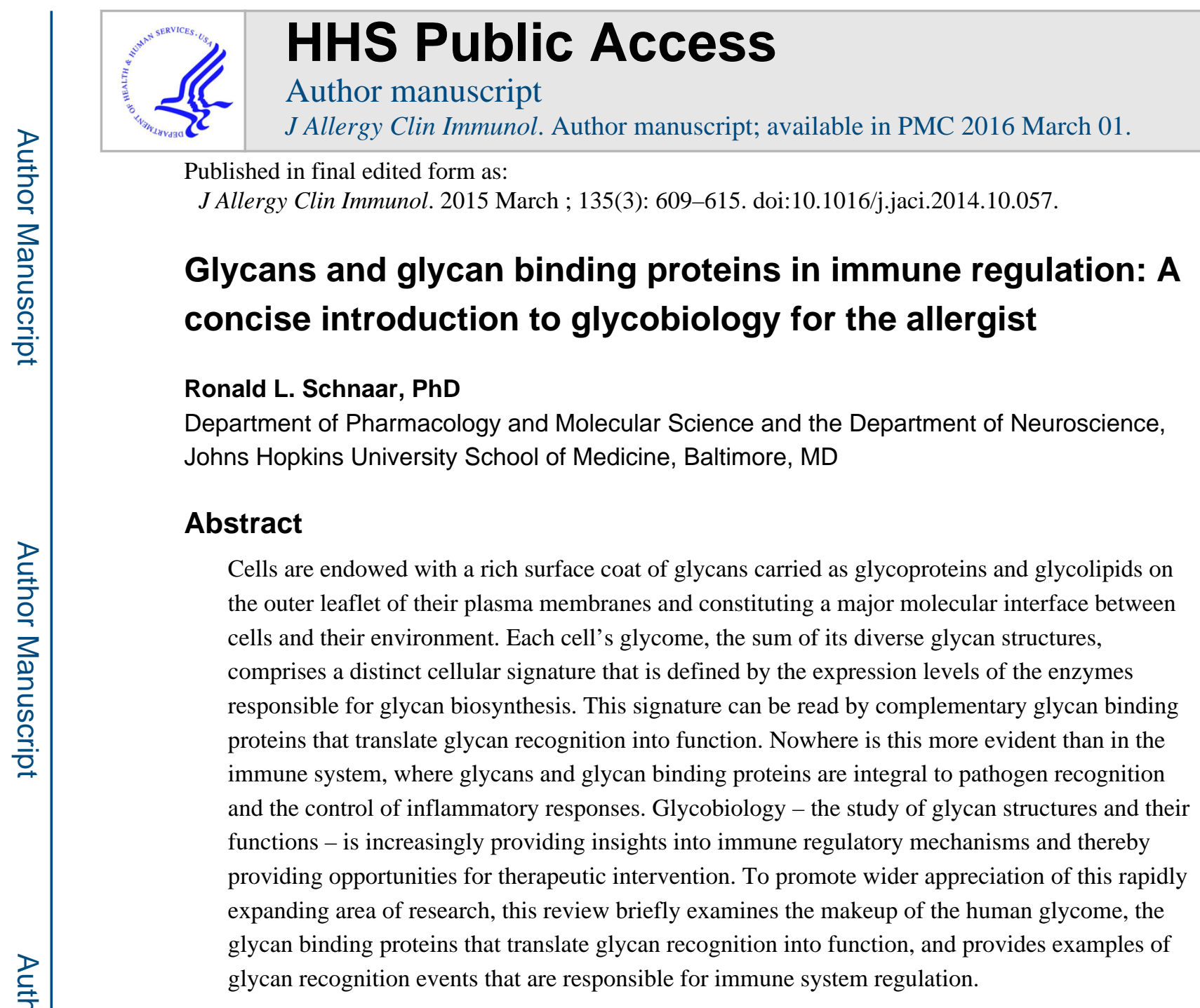

\title{
Keywords
}

glycome; lectin; selectin; galectin; siglec; sialic acid; inflammation

Cells are composed of four major classes of molecules: nucleic acids, proteins, glycans and lipids. Among these, glycans inhabit a special niche at cell surfaces, were they are involved in intermolecular and cell-cell recognition events that define and control cell interactions and functions. ${ }^{1,2}$ Glycan recognition is often mediated by complementary glycan-binding proteins (GBPs), each of which carries a specific carbohydrate-recognition domain that confers glycan binding specificity. ${ }^{3}$ Other functional domains on GBPs translate glycan binding into appropriate cellular responses. Nowhere is this more evident than in the immune system, where glycans and GBPs on the surfaces of immune cells are involved in an evolutionary chess match of immune activation and regulation within a dynamic pathogen landscape. ${ }^{2,4-6}$

Humans have $>80$ different GBPs (also known as lectins) in at least 12 structural families (see http://www.imperial.ac.uk/research/animallectins). Knowledge of the glycans and GBPs that underlie and regulate immune function provides previously unanticipated opportunities for rational design of targeted therapies for immune dysfunction, some of which show promise in the clinic. ${ }^{7,8}$ This review introduces the key players in human glycobiology: the 
human glycome (the totality of human glycan structures) and GBP families that decipher the glycan code. Examples of the roles of glycans and GBPs in infectious disease, inflammation, and the control of immune responses follow. An accompanying review in this issue provides a more focused perspective of the roles of glycans and GBPs in allergic diseases. ${ }^{9}$

\section{THE HUMAN GLYCOME}

Every cell in nature has a rich and diverse surface glycan coat that constitutes its interface with its environment. ${ }^{2}$ Whereas the complexity and diversity of glycans throughout nature are truly daunting, human glycans are more circumscribed and amenable to structurefunction studies using rapidly improving analytical techniques. A basic understanding of the building blocks, major structural themes, and general biosynthetic machinery of the human glycome provides the context for understanding glycomic regulation in the immune system.

The human glycome is built primarily from just nine monosaccharide building blocks (Fig 1), each of which is a six-membered ring (five carbons and oxygen) that is further defined by the identity and stereochemistry of the molecular constituents on each ring carbon (mostly hydroxyl groups). Monosaccharides are enzymatically linked together to form linear and branched oligosaccharides on protein and lipid carriers. Because each monosaccharide can link to any of up to four hydroxyls on another monosaccharide in one of two configurations ( $\alpha$ or $\beta$ ), and in either linear or branched arrays, an impressive diversity of distinct structures can be created from just a few building blocks. Whereas three different amino acids can combine to form six distinct tripeptides, three different monosaccharides can combine to form over a thousand distinct trisaccharides. The diversity of glycan structures found on cells is not random, but is carefully controlled by gene expression. Each cell's glycome is defined by the expression of the genes responsible for glycan biosynthesis. ${ }^{10}$ These include genes coding glycosyltransferases ( 200 in humans), glycosidases, glycan precursor biosynthetic enzymes and transporters that together represent $>3 \%$ of all human genes. Cell-specific expression of a distinct suite of glycan biosynthetic genes generates each cell's glycan "persona," a face to the world that regulates its intra- and intercellular molecular interactions. The cell's glycome varies among cell types, during differentiation, and in response to outside stimuli, providing a rich layer of regulation of cellular functions.

It is estimated that human glycosyltransferases create $\sim 7,000$ potential terminal glycan binding determinants, the basic unit of glycan recognition by GBPs (up to 5-6 sugars in a specific grouping). ${ }^{11}$ Since oligosaccharide chains are hydrophilic and often charged, they spread out in space and even minor changes, such as linkage position, linkage configuration or even the stereochemistry of a single hydroxyl group, provide the basis for the specificity of GBP recognition and downstream functional consequences. The interplay between glycan biosynthetic gene expression, glycan structure, GBP recognition, and biological function is the focus of the field of glycobiology.

Glycans at cell surfaces are classified as glycolipids or glycoproteins (Fig 1). Glycolipids have their hydrophobic lipid tail firmly embedded in the outer leaflet of the plasma membrane with their hydrophilic oligosaccharide chain extending out into the extracellular 
space. In most cells, glycolipids comprise a small percentage of total plasma membrane lipids. In contrast, nearly all proteins at the cell surface are glycoproteins, including singleand multi-pass transmembrane and secreted proteins. Glycans on proteins are classified based on their covalent linkage to the polypeptide: to an asparagine (N-linked) or to a serine or threonine (O-linked). $\mathrm{N}$-Linked protein glycosylation is initiated during protein translation in the endoplasmic reticulum whereas O-linked glycosylation occurs after translation, in the Golgi apparatus. In both cases the glycans are further modified and elaborated in the Golgi apparatus on their way to the cell surface. Whereas O-linked glycans are built stepwise and can be as small as a single sugar, N-linked glycans are pre-built on a special lipid carrier and are transferred as a characteristic 14-sugar block onto the nacient polypeptide where it is trimmed and then further elaborated. Most mature O-linked oligosaccharides are small whereas $\mathrm{N}$-linked glycans are larger. A study of the adult human lung glycome (http://www.functionalglycomics.org/) revealed that the most abundant Olinked glycans have 2-6 sugar residues as linear or short singe-branched oligosaccharides. In contrast, the major human lung N-linked glycans have 10-20 residues, most as highly branched structures (typically 3-5 branches).

In addition to $\mathrm{N}$ - and O-linked oligosaccharides, glycoproteins can also carry very long (up to $\sim 100$ sugars) repeating disaccharides linked to serine and threonine residues. These glycoproteins are classified as a separate family, the proteoglycans, to reflect their special structural features. The best known proteoglycans are heparan and chondroitin sulfates. Their long anionic sugar chains, termed glycosaminoglycans, are further substituted with sulfate groups making them even larger, more charged, and more structurally diverse. One other member of the glycosaminoglycan family, hyaluronic acid, has the same repeating sugar units but is not attached to a protein, remains unsulfated, and reaches molecular weights of millions. Proteoglycans and glycosaminoglycans, fascinating molecules deserving of their own review in the control of immune responses, are not addressed further here. $^{12}$

Glycan recognition by GBPs is most often focused on the outer-most grouping of sugars on a glycoprotein or glycolipid oligosaccharide. In humans (and other mammals), oligosaccharides are often terminated with sialic acid, the largest and most structurally complex of the monosaccharides that make up mammalian glycans (Fig 1). Evolution has often utilized sialic acid's structural complexity in developing specificity for GBP recognition. ${ }^{13}$ This is true for some pathogens, which use sialic acid to gain access to host cells, and for the immune system, which uses sialic acid for immune cell recognition, trafficking and regulation. Examples of both sialic acid dependent and independent GBPs and their roles in immune system function follow.

\section{GLYCAN BINDING PROTEINS}

Since glycans are dominant chemical determinants on the surfaces of every cell of every living organism, the glycan binding proteins that translate glycan recognition into function are also found throughout biology, from viral hemagglutinins to bacterial adhesins to plant and animal lectins. ${ }^{3}$ Every GBP is characterized by a unique carbohydrate recognition domain with precisely spaced amino acid functional groups that selectively engage a 
particular glycan target. The carbohydrate recognition domain is found in association with other protein domains that translate recognition into appropriate biological responses. Understanding glycan binding proteins, their targets, and their functions provides insight into molecular processes in immune regulation that are amenable to therapeutic intervention.

Among the first GBPs discovered were viral hemagglutinins, with influenza virus remaining the clearest example. When exposed to cultured influenza virus, human red blood cells agglutinated in a manner that spontaneously reversed over time. ${ }^{14}$ The virus-treated red blood cells remained intact, but after reversal of agglutination they were resistant to agglutination with fresh virus. The virus receptor on the red blood cell had been removed. Eventually, the factor released from red blood cells after incubation with virus was identified as sialic acid. The viral hemagglutinin was identified as a sialic acid-specific GBP, and the viral enzyme that removed the receptor was identified as a sialidase (also called neuraminidase). ${ }^{15}$ Influenza viruses bind to sialic acid-terminated oligosaccharides on the human airway epithelium, enter cells and replicate, then bud out in large numbers. The slower acting sialidase then comes into play by removing the sialic acids on the budded virus particles themselves, allowing them to disengage, disseminate, and bind to sialic acids on fresh target cells to continue the replication cycle. The rational design of sialic acid analogs (sialomimetics) able to inhibit sialidase and break this cycle led to two first-line anti-influenza drugs, Relenza ${ }^{\circledR}$ and Tamiflu ${ }^{\circledR} \cdot 16,17$ In the presence of these drugs sialic acids on the surfaces of freshly budded virions persist and engage the hemagglutinin on adjacent virions so that they clump together and fail to disseminate, halting the ongoing infection.

The carbohydrate binding domain of influenza hemagglutinin is specific for the particular way in which a terminal sialic acid is linked to the rest of the oligosaccharide chain. Avian influenza virus hemagglutinins bind to sialic acids linked to the 3-position hydroxyl of a galactose, whereas human influenza virus hemagglutinins bind to sialic acid linked to the 6position hydroxyl of galactose. ${ }^{18}$ While this seems like a modest structural difference, the shift from binding 3- to 6-linked sialic acid marks its transition from a deadly bird disease to a deadly human disease, and the Center for Disease Control is using that difference to track the potential conversion of avian to human influenza viruses worldwide. ${ }^{19}$ Many other viral, bacterial and parasitic pathogens also use glycans as adhesive targets, the study of which may provide insights into their pathophysiology and provide new antimicrobial drugs. ${ }^{20}$

Evolution has retained abundant glycans on all human cells despite the potent pathogens that take advantage of them, demonstrating their fundamental physiological roles. The first human GBP was discovered serendipitously in the late 1960's. In the intervening years $>80$ human GBPs representing a dozen different structural families have been discovered (see http://www.imperial.ac.uk/research/animallectins). While they have been found to play diverse roles, their expression and functions in the immune system continue to garner the most attention. 4,5,21,22 Examples from three families demonstrating different ways in which glycans control immunity will be presented here: galectins, selectins (a subclass of C-type lectins) and siglecs (Fig. 2). A general description of each of these GBP families is followed by specific examples of the roles of selected family members in immune system function. 
Galectins are a family of evolutionarily conserved small soluble secreted GBPs (10 in humans) with wide tissue distribution and a variety of regulatory effects on innate and adaptive immune responses. ${ }^{23}$ As their name implies, they are galactose-binding lectins, and their major glycan targets are galactose-terminated oligosaccharides on a subset of glycoproteins and glycolipids. Galectins spontaneously form dimers and higher oligomers that crosslink target glycans on cell surfaces, altering their residency time, distribution, and downstream signaling. By selectively crosslinking appropriate glycans on immune cell surface glycoproteins such as the T cell receptor, CD45 and CD43 (among others), galectins direct immune cell maturation, survival, and activation. ${ }^{24,25}$

The selectins (E-selectin, L-selectin and P-selectin) are a subset of the larger calciumdependent (C-type) lectin family. ${ }^{26}$ They are single-pass transmembrane cell adhesion proteins, each with an externally-facing carbohydrate recognition domain that binds to a grouping of sugars that include appropriately spaced sialic acid and fucose residues. E- and P-selectins are expressed on vascular endothelial cells in response to inflammatory signals and then bind to glycans on passing leukocytes to initiate inflammation. L-Selectin is expressed on leukocytes and binds to glycans on the lumen of high endothelial venules, initiating migration of leukocytes from blood to lymph. The three selectins are examples of sub-specialization within the diverse C-type lectin family, which includes other important immune regulatory lectins such as the mannose-binding lectin, DC-SIGN, Dectins, and Mincle (among others). ${ }^{27}$

Siglecs (sialic acid binding immunoglobulin like lectins) are the most recently discovered family of human glycan binding proteins. ${ }^{21}$ The family is comprised of 14 members in humans, most of which are selectively expressed on subsets of hemopoietic cells. They are single-pass transmembrane proteins with varying numbers of externally facing Ig-like domains terminated with a carbohydrate recognition Ig-like domain that binds to sialic acidterminated glycans. Many members also have immune regulatory sequences (ITIMs or ITAMs) on their cytoplasmic tails, indicating their roles in immune regulatory transmembrane signaling. Although every siglec requires a sialic acid in its glycan binding target, the family has evolved to take advantage of the many ways in which sialic acids are arranged in larger glycan structures. Like the influenza virus hemagglutinin, some siglecs bind only to sialic acid when it is linked to the 3-hydroxyl of galactose and others only to sialic acid linked to the 6-hydroxyl of galactose. Yet others prefer sialic acid linked to the 8hydroxyl of another sialic acid, and some require other spaced molecular constituents (such as sulfates) on their oligosaccharide targets. In a metaphorical sea of cell surface sialic acids, these glycan binding specificities provide selective recognition to siglec family members that are key to their functions in immune regulation.

\section{GLYCANS AND GLYCAN BINDING PROTEINS IN IMMUNE REGULATION}

GBPs, which are expressed on immune cells of all types, serve diverse regulatory rolls in leukocyte trafficking, pathogen recognition, antigen processing, immune activation, and immunosuppression. ${ }^{4,5,22}$ Glycans on immune cells are also recognized by GBPs on nonimmune cells and tissues (like those on the vascular endothelium) that bind to leukocyte glycans. The three vignettes from this extensive area of research that follow (Fig. 2) 
demonstrate paradigms for glycan-mediated immune regulation. Additional examples that are especially relevant to allergic diseases are detailed in an accompanying paper in this volume. ${ }^{9}$

\section{Galectin binding to $\mathrm{N}$-glycans on the $\mathrm{T}$ cell receptor tunes adaptive immune responses}

The adaptive immune response is carefully tuned to protect against autoimmunity. One mechanism by which this occurs is via galectins binding to $\mathrm{N}$-linked glycans on the $\mathrm{T}$ cell receptor (TCR) ${ }^{28,29}$ As glycoproteins, including the TCR, traverse the Golgi apparatus their nacient $\mathrm{N}$-linked glycans are subject to modification by a suite of glycosidases and glycosyltransferases that act in concert to generate mature glycans on selected protein targets. The TCR (along with many other glycoproteins) is subject to the action of the glycosyltransferase "mannoside $\mathrm{N}$-acetylglucosaminyltransferase 5," the product of the MGAT5 gene. This leads to elongation of one of its N-linked glycan branches with repeated Gal-GlcNAc repeats, which are high-affinity receptors for galectins such as Galectin-3. Once the TCR reaches the surface of T cells, the Gal-GlcNAc repeats on the TCR recruit galectins, which crosslink the TCRs into a cell surface lattice that restricts the TCR clustering required for $\mathrm{T}$ cell activation. The effect of galectin-TCR lattice formation is that the $\mathrm{T}$ cells require higher agonist concentrations to trigger activation. Thus the action of a particular Golgi glycosyltransferase in $\mathrm{T}$ cells is responsible for fine-tuning its responsiveness to agonists.

In a potential feedback loop, activation of the TCR regulates expression of Golgi glycosidases and glycosyltransferases to shift the balance of N-linked glycans on the TCR toward those with enhanced galectin binding. ${ }^{30}$ In this way, initial TCR activation may be followed by glycan-regulated desensitization.

The function of this pathway in immune regulation was demonstrated in Mgat5-knockout mice, which display worse outcomes (enhanced sensitivity) in models of autoimmunity including autoimmune glomerulonephritis, delayed-type hypersensitivity, and autoimmune encephalomyelitis (EAE, an animal model for multiple sclerosis). ${ }^{28}$ In humans, genome wide association studies revealed hypomorphic MGAT5 variants as factors in multiple sclerosis susceptibility. ${ }^{31}$ Remarkably, oral administration of $\mathrm{N}$-acetylglucosamine to mice increased the galectin-binding $\mathrm{N}$-glycan structures on their T cells, reduced TCR signaling and reduced the symptoms of experimental autoimmunity. ${ }^{32}$ This $\mathrm{N}$-glycan link to $\mathrm{T}$ cell activation may provide new ways to intervene in human autoimmune diseases.

\section{Selectins initiate leukocyte extravasation during inflammation}

Cell adhesion between circulating leukocytes and the vascular endothelium initiates leukocyte movement across the vascular wall into tissues. Glycans and GBPs, notably glycans on leukocytes that bind to E-selectin and P-selectin on the endothelium, initiate this process. ${ }^{33}$ Leukocytes typically ignore the blood vessel wall until inflammatory signals (e.g. histamine, bacterial lipopolysaccharides, TNF-a) activate the expression of selectins by vascular endothelial cells. P-selectin is pre-packaged in granules (Weibel-Palade bodies) inside the endothelial cells ready for rapid deployment to the cell surface within minutes of detection of an inflammatory signal, whereas E-selectin is expressed over a longer time 
frame. Surface-expressed E- and P-selectin snag passing leukocytes from the blood by binding to pre-existing leukocyte cell surface glycans. The leukocytes then roll along the endothelium before stably adhering and migrating into the adjoining tissues.

E- and P-selectin bind to related but distinct glycans on human neutrophils. E-Selectin binds to sialylated fucosylated glycolipids, ${ }^{34}$ whereas P-selectin binds to sialylated fucosylated Olinked glycans on the glycoprotein PSGL-1. ${ }^{26}$ The commonality of fucose as an essential part of the target glycans for selectins leads to the human leukocyte adhesion deficiency LAD-II. ${ }^{35,36}$ This rare congenital disorder results in insufficient addition of fucose to glycans. Affected individuals are unable to mount inflammatory responses and suffer repeated and persistent tissue bacterial infections despite high circulating leukocyte counts. Remarkably, oral administration of fucose can dramatically reverse this phenotype, leading to effective leukocyte extravasation and re-establishment of inflammatory anti-bacterial responses. The underlying genetic fault is in a transporter (coded by the $S L C 35 \mathrm{Cl}$ gene) that carries an activated form of fucose from the cytoplasm into the Golgi apparatus where it is required for fucosyltransferase-mediated synthesis of selectin-binding glycans. ${ }^{37,38} \mathrm{By}$ administering oral fucose, the concentration of activated fucose entering the Golgi apparatus increases sufficiently to reinstate selectin ligand biosynthesis thereby reinstating selectinmediated leukocyte adhesion. These clinical observations provide convincing evidence of the essential role of glycans and GBPs in cell-cell adhesion in the immune system and in effective immune system function.

Based on the structures of glycans bound to selectins, a pan-selectin inhibitor, Rivipansel (GMI-1070), was developed and has completed initial trails for vaso-occlusive crisis, an inflammatory disorder resulting in significant morbidity in Sickle Cell patients. ${ }^{8,39}$

Rivipansel retains an intact fucose residue, a sialic acid mimetic, an $\mathrm{N}$-acetylglucosamine mimetic and a functionalized galactose. It is a model for the potential of glycobiology to provide therapeutic lead molecules to target glycan-mediated processes. ${ }^{7}$

\section{Siglec-1 (sialoadhesin) balances self and non-self recognition in adaptive immunity}

Of the 14 human siglecs, 13 are expressed on more or less restricted sets of cells of the hemopoietic lineage. ${ }^{21}$ The result is overlapping expression of siglecs throughout the innate and adaptive immune systems. The immune functions of siglecs depend on the cells on which they are expressed and the roles of those cells in physiology and pathology. Examples relevant to lung inflammatory diseases are discussed in more detail in an accompanying review in this issue. ${ }^{9}$ Siglecs function by binding to sialoglycans on their own cell surfaces, on other cells and tissues, and/or on sialic acid-bearing pathogens. ${ }^{21}$ The interplay of sialic acids and sialic acid recognition in immune regulation, pathogenesis, and pathogen recognition results in something of an evolutionary arms race as pathogens and hosts respond to changing glycan landscapes. ${ }^{40}$

Siglec-1 is a major receptor on macrophages and plays important roles in both self and pathogen recognition and responses. ${ }^{41}$ It is well conserved in mammals where it is restricted to macrophages, especially those in lymph nodes and spleen residing at the borders of lymphoid tissues and circulating fluids where they come in contact with both blood-borne pathogens and lymphocytes. Siglec-1 is notable for its size (17 Ig-like domains) and shape, 
with a globular head attached to a long stalk inserted in the membrane with a short cytoplasmic tail. This places the N-terminal carbohydrate recognition domain well above the cell surface where it interacts with extracellular targets including other cells and pathogens. Its sialoglycan binding site on the outermost Ig-like domain is a shallow pocket that cradles the sialic acid and adjoining sugars against precisely spaced amino acids that provide ionic, hydrogen, and hydrophobic bonds that cooperate to specifically engage glycans bearing terminal a2,3-linked sialic acids. ${ }^{42}$

Siglec-1 provides a mechanism for binding, clearance, and antigen presentation of pathogenic bacteria. Although sialic acid is not broadly found in prokaryotes, several important human pathogens express sialoglycans, some with structures quite similar to those in humans. ${ }^{43}$ Sialic acid-expressing pathogens include group B Streptococcus (GBS), N. meningitides, Campylobacter jejuni, Haemaphilus influenza, and pathogenic E. coli. The finding that Siglec-1 is sharply upregulated during infection, especially via the type I interferon pathway, suggested it may play a role in responses to sialylated pathogens. ${ }^{44}$ This has been confirmed in vivo. In response to intravenous injection of GBS, wild type mice effectively cleared the pathogen into secondary lymphoid tissue macrophages, whereas mice lacking Siglec-1 suffered increased pathogen dissemination and decreased survival. ${ }^{45}$ Likewise, injected $C$. jejuni were more effectively cleared into spleen macrophages in wild type compared to mice lacking functional Siglec-1, and capture was accompanied by a robust proinflammatory response. ${ }^{46}$

In addition to clearing and killing pathogens, Siglec-1 processes and presents antigens to B cells and invariant natural killer T cells (iNKT) to enhance adaptive immunity. ${ }^{47,48}$ This pathway has been accessed experimentally to improve the delivery of protein and lipid antigens to the immune system. ${ }^{49,50}$ In one study, a nanoparticle decorated with a sialomimetic that selectively binds Siglec-1 delivered an iNKT-activating lipid to macrophages, which then presented the antigen to iNKT cells (via CD1d) leading to an enhanced inflammatory response. 50

Despite its useful functions in immune surveillance and response, Siglec-1 also facilitates disease progression both by pathogen binding and lymphocyte dysregulation. Enveloped viruses that carry sialic acids on their surfaces, such as HIV, use Siglec-1 to gain access to macrophage intracellular compartments, thereby promoting trans-infection and dissemination to T cells. ${ }^{51}$ Siglec- 1 can also exacerbate autoimmunity, at least in animal models. ${ }^{52}$ The expansion of regulatory T cells (Tregs), a process important to immune system balance, is directly inhibited when Siglec-1 binds to abundant sialoglycan targets on Treg surfaces, thereby shifting the immune response toward pro-inflammation. In animal models of nervous system autoimmunity, Siglec-1-null mice experienced reduced inflammatory responses with reduced disease progression in every case, implicating Siglec-1 as a facilitating factor in the progression of autoimmunity. 44

The above examples are representative of the many glycan-mediated recognition and regulation events important for proper immune system function. They represent the functions of glycans and GBPs in immune activation and in moderation of the immune response in health and disease. As the field continues to grow and develop enhanced tools 
for glycan synthesis, analysis, and function, glycobiology promises to provide previously unanticipated insights and opportunities for therapeutic intervention.

\section{Acknowledgments}

Supported by a program project grant from the National Institutes of Health/National Heart, Lung, and Blood Institute (P01 HL-107151)

\section{Abbreviations used}

$\begin{array}{ll}\text { GBP } & \text { glycan binding protein } \\ \text { TCR } & \text { T cell receptor } \\ \text { GBS } & \text { group B Streptococcus } \\ \text { iNKT } & \text { invariant natural killer T cells }\end{array}$

\section{References}

1. Paulson JC, Blixt O, Collins BE. Sweet spots in functional glycomics. Nat Chem Biol. 2006; 2:238248. [PubMed: 16619023]

2. Varki A. Evolutionary forces shaping the Golgi glycosylation machinery: why cell surface glycans are universal to living cells. Cold Spring Harb Perspect Biol. 2011; 3:a005462. [PubMed: 21525513]

3. Taylor ME, Drickamer K. Convergent and divergent mechanisms of sugar recognition across kingdoms. Curr Opin Struct Biol. 2014; 28C:14-22. [PubMed: 25102772]

4. Marth JD, Grewal PK. Mammalian glycosylation in immunity. Nat Rev Immunol. 2008; 8:874-887. [PubMed: 18846099]

5. van Kooyk Y, Rabinovich GA. Protein-glycan interactions in the control of innate and adaptive immune responses. Nat Immunol. 2008; 9:593-601. [PubMed: 18490910]

6. Rabinovich GA, van KY, Cobb BA. Glycobiology of immune responses. Ann N Y Acad Sci. 2012; 1253:1-15. [PubMed: 22524422]

7. Ernst B, Magnani JL. From carbohydrate leads to glycomimetic drugs. Nat Rev Drug Discov. 2009; 8:661-677. [PubMed: 19629075]

8. Wun T, Styles L, DeCastro L, Telen MJ, Kuypers F, Cheung A, et al. Phase 1 study of the E-selectin inhibitor GMI 1070 in patients with sickle cell anemia. PLoS ONE. 2014; 9:e101301. [PubMed: 24988449]

9. Bochner BS, Zimmermann N. Role of siglecs and related glycan-binding proteins in immune responses and immunoregulation. J Allergy Clin Immunol. 2014; 135 in press.

10. Nairn AV, York WS, Harris K, Hall EM, Pierce JM, Moremen KW. Regulation of glycan structures in animal tissues: transcript profiling of glycan-related genes. J Biol Chem. 2008; 283:17298-17313. [PubMed: 18411279]

11. Cummings RD. The repertoire of glycan determinants in the human glycome. Mol Biosyst. 2009; 5:1087-1104. [PubMed: 19756298]

12. Frey H, Schroeder N, Manon-Jensen T, Iozzo RV, Schaefer L. Biological interplay between proteoglycans and their innate immune receptors in inflammation. FEBS J. 2013; 280:2165-2179. [PubMed: 23350913]

13. Cohen M, Varki A. The sialome--far more than the sum of its parts. OMICS. 2010; 14:455-464. [PubMed: 20726801]

14. Hirst GK. Adsorption of influenza hemagglutinins and virus by red blood cells. J Exp Med. 1942; 76:195-209. [PubMed: 19871229]

15. Gottschalk A. The influenza virus neuraminidase. Nature. 1958; 181:377-378. [PubMed: 13504207] 
16. von Itzstein M, Wu WY, Kok GB, Pegg MS, Dyason JC, Jin B, et al. Rational design of potent sialidase-based inhibitors of influenza virus replication. Nature. 1996; 363:418-423. [PubMed: 8502295]

17. von Itzstein M. The war against influenza: discovery and development of sialidase inhibitors. Nat Rev Drug Discov. 2007; 6:967-974. [PubMed: 18049471]

18. Stevens J, Blixt O, Paulson JC, Wilson IA. Glycan microarray technologies: tools to survey host specificity of influenza viruses. Nat Rev Microbiol. 2006; 4:857-864. [PubMed: 17013397]

19. Stevens J, Blixt O, Chen LM, Donis RO, Paulson JC, Wilson IA. Recent avian H5N1 viruses exhibit increased propensity for acquiring human receptor specificity. J Mol Biol. 2008; 381:1382-1394. [PubMed: 18672252]

20. Bernardi A, Jimenez-Barbero J, Casnati A, De CC, Darbre T, Fieschi F, et al. Multivalent glycoconjugates as anti-pathogenic agents. Chem Soc Rev. 2013; 42:4709-4727. [PubMed: 23254759]

21. Macauley MS, Crocker PR, Paulson JC. Siglec-mediated regulation of immune cell function in disease. Nat Rev Immunol. 2014; 14:653-666. [PubMed: 25234143]

22. Johnson JL, Jones MB, Ryan SO, Cobb BA. The regulatory power of glycans and their binding partners in immunity. Trends Immunol. 2013; 34:290-298. [PubMed: 23485517]

23. Liu FT, Rabinovich GA. Galectins: regulators of acute and chronic inflammation. Ann N Y Acad Sci. 2010; 1183:158-182. [PubMed: 20146714]

24. Garner OB, Baum LG. Galectin-glycan lattices regulate cell-surface glycoprotein organization and signalling. Biochem Soc Trans. 2008; 36:1472-1477. [PubMed: 19021578]

25. Rabinovich GA, Toscano MA. Turning 'sweet' on immunity: galectin-glycan interactions in immune tolerance and inflammation. Nat Rev Immunol. 2009; 9:338-352. [PubMed: 19365409]

26. Somers WS, Tang J, Shaw GD, Camphausen RT. Insights into the molecular basis of leukocyte tethering and rolling revealed by structures of P- and E-selectin bound to SLe(X) and PSGL-1. Cell. 2000; 103:467-479. [PubMed: 11081633]

27. Hoving JC, Wilson GJ, Brown GD. Signalling C-type lectin receptors, microbial recognition and immunity. Cell Microbiol. 2014; 16:185-194. [PubMed: 24330199]

28. Demetriou M, Granovsky M, Quaggin S, Dennis JW. Negative regulation of T-cell activation and autoimmunity by Mgat5 N-glycosylation. Nature. 2001; 409:733-739. [PubMed: 11217864]

29. Dennis JW, Lau KS, Demetriou M, Nabi IR. Adaptive regulation at the cell surface by Nglycosylation. Traffic. 2009; 10:1569-1578. [PubMed: 19761541]

30. Chen HL, Li CF, Grigorian A, Tian W, Demetriou M. T cell receptor signaling co-regulates multiple Golgi genes to enhance N-glycan branching. J Biol Chem. 2009; 284:32454-32461. [PubMed: 19706602]

31. Li CF, Zhou RW, Mkhikian H, Newton BL, Yu Z, Demetriou M. Hypomorphic MGAT5 polymorphisms promote multiple sclerosis cooperatively with MGAT1 and interleukin-2 and 7 receptor variants. J Neuroimmunol. 2013; 256:71-76. [PubMed: 23351704]

32. Grigorian A, Lee SU, Tian W, Chen IJ, Gao G, Mendelsohn R, et al. Control of T Cell-mediated autoimmunity by metabolite flux to N-glycan biosynthesis. J Biol Chem. 2007; 282:20027-20035. [PubMed: 17488719]

33. Zarbock A, Ley K, McEver RP, Hidalgo A. Leukocyte ligands for endothelial selectins: specialized glycoconjugates that mediate rolling and signaling under flow. Blood. 2011; 118:6743-6751. [PubMed: 22021370]

34. Nimrichter L, Burdick MM, Aoki K, Laroy W, Fierro MA, Hudson SA, et al. E-selectin receptors on human leukocytes. Blood. 2008; 112:3744-3752. [PubMed: 18579791]

35. Marquardt T, Luhn K, Srikrishna G, Freeze HH, Harms E, Vestweber D. Correction of leukocyte adhesion deficiency type II with oral fucose. Blood. 1999; 94:3976-3985. [PubMed: 10590041]

36. Luhn K, Marquardt T, Harms E, Vestweber D. Discontinuation of fucose therapy in LADII causes rapid loss of selectin ligands and rise of leukocyte counts. Blood. 2001; 97:330-332. [PubMed: 11133780]

37. Luhn K, Wild MK, Eckhardt M, Gerardy-Schahn R, Vestweber D. The gene defective in leukocyte adhesion deficiency II encodes a putative GDP-fucose transporter. Nat Genet. 2001; 28:69-72. [PubMed: 11326279] 
38. Hellbusch CC, Sperandio M, Frommhold D, Yakubenia S, Wild MK, Popovici D, et al. Golgi GDP-fucose transporter-deficient mice mimic congenital disorder of glycosylation IIc/leukocyte adhesion deficiency II. J Biol Chem. 2007; 282:10762-10772. [PubMed: 17276979]

39. Chang J, Patton JT, Sarkar A, Ernst B, Magnani JL, Frenette PS. GMI-1070, a novel pan-selectin antagonist, reverses acute vascular occlusions in sickle cell mice. Blood. 2010; 116:1779-1786. [PubMed: 20508165]

40. Padler-Karavani V, Hurtado-Ziola N, Chang YC, Sonnenburg JL, Ronaghy A, Yu H, et al. Rapid evolution of binding specificities and expression patterns of inhibitory CD33-related Siglecs in primates. FASEB J. 2014; 28:1280-1293. [PubMed: 24308974]

41. Klaas M, Crocker PR. Sialoadhesin in recognition of self and non-self. Semin Immunopathol. 2012; 34:353-364. [PubMed: 22450957]

42. May AP, Robinson RC, Vinson M, Crocker PR, Jones EY. Crystal structure of the N-terminal domain of sialoadhesin in complex with 3' sialyllactose at 1.85 A resolution. Mol Cell. 1998; 1:719-728. [PubMed: 9660955]

43. Severi E, Hood DW, Thomas GH. Sialic acid utilization by bacterial pathogens. Microbiology. 2007; 153:2817-2822. [PubMed: 17768226]

44. O'Neill AS, van den Berg TK, Mullen GE. Sialoadhesin - a macrophage-restricted marker of immunoregulation and inflammation. Immunology. 2013; 138:198-207. [PubMed: 23181380]

45. Chang YC, Olson J, Louie A, Crocker PR, Varki A, Nizet V. Role of macrophage sialoadhesin in host defense against the sialylated pathogen group B Streptococcus. J Mol Med (Berl). 2014; 92:951-959. [PubMed: 24788876]

46. Klaas M, Oetke C, Lewis LE, Erwig LP, Heikema AP, Easton A, et al. Sialoadhesin promotes rapid proinflammatory and type I IFN responses to a sialylated pathogen, Campylobacter jejuni. J Immunol. 2012; 189:2414-2422. [PubMed: 22851711]

47. Junt T, Moseman EA, Iannacone M, Massberg S, Lang PA, Boes M, et al. Subcapsular sinus macrophages in lymph nodes clear lymph-borne viruses and present them to antiviral B cells. Nature. 2007; 450:110-114. [PubMed: 17934446]

48. Barral P, Polzella P, Bruckbauer A, van RN, Besra GS, Cerundolo V, et al. CD169(+) macrophages present lipid antigens to mediate early activation of iNKT cells in lymph nodes. Nat Immunol. 2010; 11:303-312. [PubMed: 20228797]

49. Delputte PL, Van GH, Favoreel HW, Hoebeke I, Delrue I, Dewerchin H, et al. Porcine sialoadhesin (CD169/Siglec-1) is an endocytic receptor that allows targeted delivery of toxins and antigens to macrophages. PLoS ONE. 2011; 6:e16827. [PubMed: 21359217]

50. Kawasaki N, Vela JL, Nycholat CM, Rademacher C, Khurana A, van RN, et al. Targeted delivery of lipid antigen to macrophages via the CD169/sialoadhesin endocytic pathway induces robust invariant natural killer T cell activation. Proc Natl Acad Sci U S A. 2013; 110:7826-7831. [PubMed: 23610394]

51. Izquierdo-Useros N, Lorizate M, McLaren PJ, Telenti A, Krausslich HG, Martinez-Picado J. HIV-1 capture and transmission by dendritic cells: the role of viral glycolipids and the cellular receptor Siglec-1. PLoS Pathog. 2014; 10:e1004146. [PubMed: 25033082]

52. Wu C, Rauch U, Korpos E, Song J, Loser K, Crocker PR, et al. Sialoadhesin-positive macrophages bind regulatory $\mathrm{T}$ cells, negatively controlling their expansion and autoimmune disease progression. J Immunol. 2009; 182:6508-6516. [PubMed: 19414805]

53. Varki A, Cummings RD, Esko JD, Freeze HH, Stanley P, Marth JD, et al. Symbol nomenclature for glycan representation. Proteomics. 2009; 9:5398-5399. [PubMed: 19902428] 

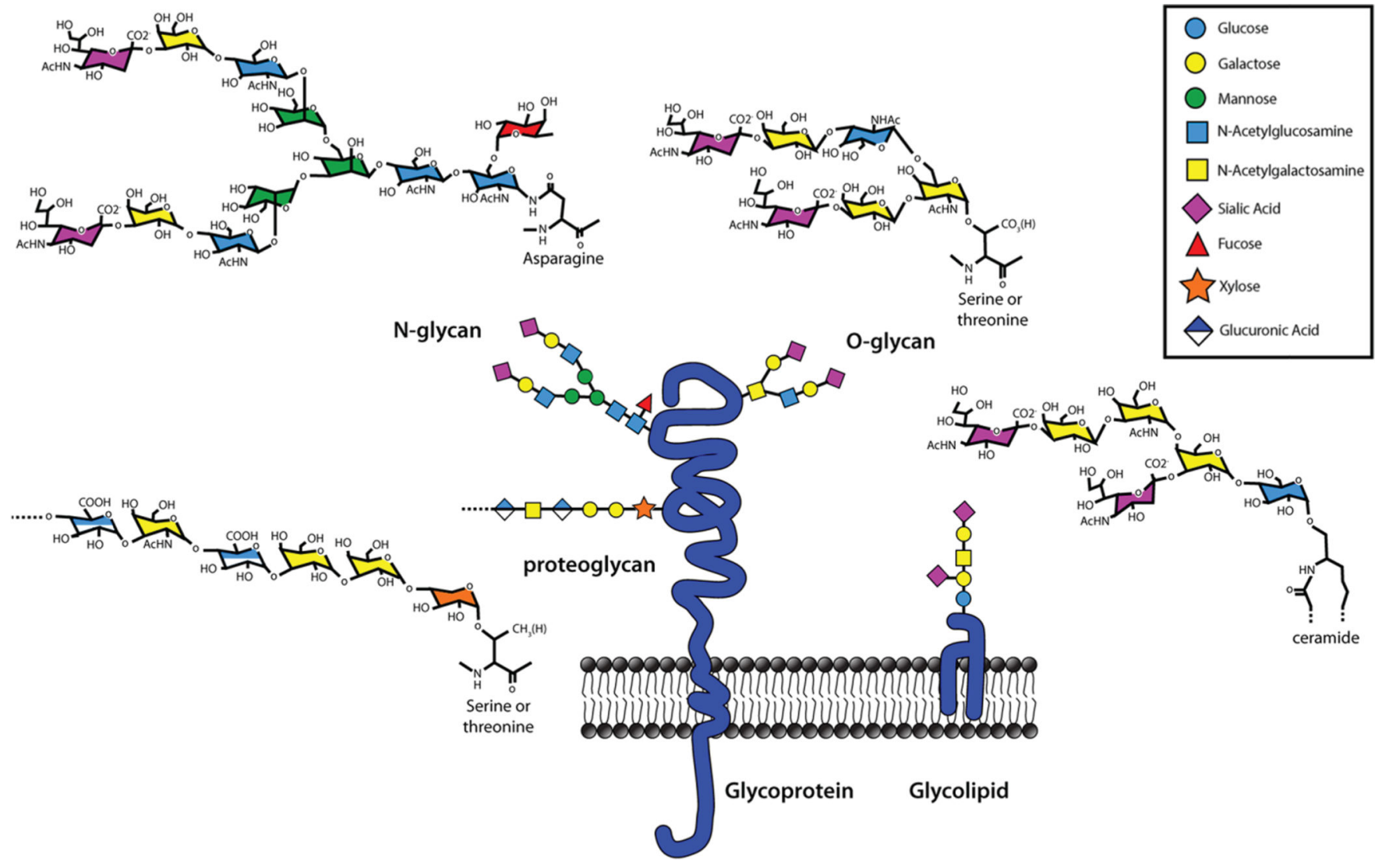

FIG. 1.

Cell surface glycans. All mammalian cells are endowed with a dense and diverse surface coat of glycans comprised of glycoproteins, proteoglycans and glycolipids. They are built primarily from just nine different monosaccharide building blocks, each a six-membered ring with distinct structural substituents. A color-coded symbol nomenclature for these (key) has been agreed upon by the field. ${ }^{53} \mathrm{~N}$-Linked glycans are attached to protein asparagine residues and are invariably branched structures that display varied termini. O-Linked glycans are attached to protein serine or threonine residues and typically consist of shorter branched or unbranched structures. Proteoglycans are also attached to protein serine or threonine residues, but are notable for their long disaccharide repeats, most of which are highly charged due to the abundance of sugar acids and sulfates. Mammalian glycolipids primarily consist of glycans attached to a ceramide lipid moiety. Together these glycans comprise the glycome of each cell, providing it with the diverse surface structures required for that cell's functions. 


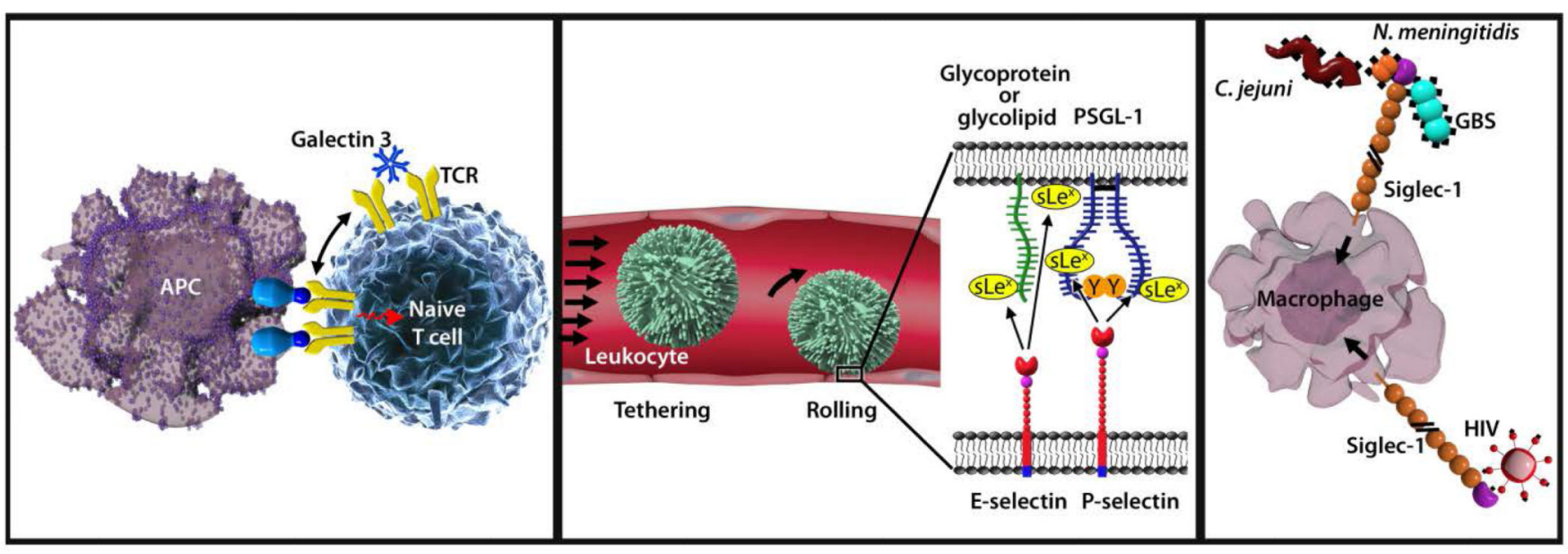

FIG. 2.

Representative roles of glycans and glycan binding proteins in immune regulation. (Left panel) Galectin-3 crosslinks T cell receptors (TCR) via their N-linked glycans to regulate immune activation. (Center panel) E- and P-Selectins engage glycans on the surface of passing leukocytes to mediate the initial (tethering) step of inflammation. (Right panel) Siglec-1 (sialoadhesin) captures sialylated pathogens for clearance and antigen presentation, but can be commandeered by HIV for trans-infection and dissemination. 